\title{
Insulinoma, presentación y evolución de dos casos clínicos
}

\author{
JUAN JAVIER LAMMOGLIA H. ${ }^{1}$, MARÍA ISABEL HERNÁNDEZ C. ${ }^{1}$, \\ NANCY UNANUE M. ${ }^{1}$, ROSSANA ROMÁN R. ${ }^{1}$, JORGE VILLANUEVA B. ${ }^{1}$, ALEJANDRA AVILA A. ${ }^{1}$, \\ VERÓNICA MERICQ G. ${ }^{1}$, ETHEL CODNER D. ${ }^{1}$, FERNANDO CASSORLA G. ${ }^{1}$
}

1. Instituto de Investigaciones Materno Infantil. Universidad de Chile, Santiago, Chile.

\begin{abstract}
Insulinoma, evolution and presentation of 2 clinical cases

Insulinoma is a very uncommon tumor in children, with an incidence in adults of 2 per million inhabitants. Clinical manifestations include neuroglycopenic or autonomic manifestations due to hypoglycemia. We describe 2 pediatric patients with insulinoma, characterized by repeated episodes of hypoglycemia associated to high insulin serum levels and presence of a small mass in the pancreas by imaging studies. The diagnosis was very prompt in one case and delayed in the other, emphasizing the need for an appropriate diagnosis of hypoglycemia during childhood.

(Key words: insulinoma, hypoglycemia, insulin, pancreas).

Rev Chil Pediatr 2008; 79 (2): 181-185

\section{RESUMEN}

El insulinoma es un tumor muy infrecuente en la edad pediátrica y la incidencia reportada en adultos es de 2 casos por millón de habitantes. La presentación de la enfermedad consiste en la presencia de síntomas neuroglicopénicos y autonómicos desencadenados por los episodios de hipoglicemia. Se describen dos pacientes con insulinoma esporádico. El cuadro clínico consistió en episodios repetidos de hipoglicemia asociados a niveles aumentados de insulina sérica y a imágenes sugerentes de un tumor pancreático. El diagnóstico fue muy oportuno en uno de los casos y muy tardío en el otro, lo que resalta la necesidad de estar muy alerta ante casos de hipoglicemia durante la niñez.

(Palabras clave: insulinoma, hipoglicemia, insulina, páncreas).

Rev Chil Pediatr 2008; 79 (2): 181-185
\end{abstract}

Trabajo recibido el 20 de noviembre de 2007, aceptado para publicación el 23 de enero de 2008.

Correspondencia a:

Fernando Cassorla G.

E-mail:fcassorl@med.uchile.cl 


\section{Introducción}

El insulinoma es un tumor muy infrecuente durante la niñez, la incidencia durante la vida adulta es de alrededor de 2 casos por millón de habitantes ${ }^{1}$. Los insulinomas son generalmente causados por tumores pequeños de alrededor de $1 \mathrm{~cm}$ de diámetro ubicados en la cabeza o unión de cabeza y cuerpo del páncreas, cerca del ducto pancreático. La presentación clínica de la enfermedad consiste en la presencia de síntomas neuroglicopénicos y autonómicos desencadenados por los episodios de hipoglicemia (tabla 1). El diagnóstico se basa en la tríada descrita por Whipple ${ }^{2}$, que consiste en presencia de alteraciones de comportamiento y/o síntomas disautonómicos, glicemia menor a 40 mg/dl y resolución del cuadro luego de aportar glucosa y normalizar los valores de glicemia.

Junto con la tríada descrita, el cuadro clínico puede revelar síntomas sutiles como aumento inusual del apetito o cambios de comportamiento asociados a los episodios de hipoglicemia, retardando el diagnóstico y manejo adecua$\mathrm{do}^{2,3}$.

La secreción exagerada de insulina determina que las vías alternativas para la utilización de la glucosa estén bloqueadas, por lo que se restringe la glucogenolisis y la liberación de ácidos grasos. Esto limita la disponibilidad de metabolitos alternativos para la nutrición del cerebro, lo que conlleva riesgo de secuelas neurológicas.

El objetivo de este trabajo es describir dos casos clínicos de insulinoma en pacientes pediátricos y discutir algunos puntos del estudio y manejo de la hipoglicemia en el niño mayor de un año.

Tabla 1. Semiología de los síntomas neuroglicopénicos y autonómicos en hipoglicemia, con énfasis en los síntomas que hacen sospechar esta patología en la edad pediátrica

- Autonómicos: Diaforesis, temblor, taquicardia, ansiedad, nerviosismo, debilidad, hambre, náuseas, vómito

- Glucopenia SNC: Cefalea, visión borrosa, letargia, irritabilidad, lenguaje alterado, somnolencia, convulsiones, disminución concentración, conductas bizarras y cambios de personalidad transitorios a permanentes
- Sospecha en pediatría:

- Inatención

- Estrabismo reciente

- Letargia-somnolencia

- Problemas de conducta

- Apetito exagerado con o sin obesidad secundaria

- Convulsiones 
terapia antimicrobiana. La paciente actualmente completa 14 meses de seguimiento, sin registro de hipoglicemia.

\section{Paciente 2}

Varón de 14 años, que a los 10 años presentó un episodio de síncope con evaluación en servicio de urgencia infantil evidenciándose una glicemia de $10 \mathrm{mg} / \mathrm{dl}$. El paciente evolucionó con episodios convulsivos frecuentes, con registro de glucometrías con valores alrededor de $50 \mathrm{mg} / \mathrm{dl}$. Sin antecedentes personales de importancia y con un desarrollo psicomotor y rendimiento escolar normales previos a los 10 años, el interrogatorio fue negativo para exposición a insulina o hipoglicemiantes orales.

Al momento de la evaluación su peso estaba en percentil 15, talla en percentil 1 y tenía un volumen testicular de $5 \mathrm{ml}$. Se indicó una prueba de ayuno la cual se suspendió a la hora por hipoglicemia severa, y cuyos resultados se describen en la tabla 2, destacando hipoglicemia de $25 \mathrm{mg} \%$ con insulinemia de $15 \mathrm{mUI} / \mathrm{ml}$ y cetonemia negativa; respuesta a glucagón positiva.

Durante la hospitalización se realizó una ecografía de páncreas que describió una masa en la unión de la cabeza con el cuerpo del páncreas de $1,5 \mathrm{~cm}$ de diámetro, confirmada por TAC abdominal y por angioresonancia. El paciente requirió manejo con infusión constante de glucosa a $8 \mathrm{mg} / \mathrm{kg} / \mathrm{min}$, asociado a un régimen de alimentación frecuente, con lo cual se controlaron los episodios de hipoglicemia. Una evaluación por neuropsiquiatría encontró un transtorno oposicional desafiante con coeficiente intelectual limítrofe y discontrol de impulsos, cuadro que no respondió a risperidona. Con diagnóstico de posible insulinoma, se realizó una pancreatoduodenectomía más colecistectomía, que evidenció una masa de $1,7 \mathrm{~cm}$ en la unión de la cabeza y el cuerpo de páncreas. La palpación cuidadosa intraoperatoria no evidenció otras lesiones. La anatomía patológica describió un tumor pancreático bien diferenciado, no encapsulado, de $1,7 \mathrm{~cm}$ de diámetro con 2 mitosis en 10 campos. En el post operatorio el paciente presentó una pancreatitis con colección intraabdominal que requirió una hospitalización de 6 semanas. Luego de la cirugía no se han detectado nuevos episodios de hipoglicemia.

\section{Discusión}

La hipoglucemia en el niño mayor hace necesario plantearse la posibilidad de cuadros tóxicos, hepáticos y más raramente cuadros endocrinos como déficits hormonales o exceso de insulina (tabla 3) ${ }^{4}$. La evaluación del paciente

Tabla 3. Causas de hipoglicemia en el niño mayor y adolescente

- Intoxicaciones Insulina, Etanol, Betabloqueadores, Glibenclamida

- Falla suprarenal - Enfermedad de Addisson

- Falla hepática aguda Hepatitis viral - Síndrome de Reye Vasculitiis

- Hipopituitarismo Craneofaringioma

- Insulinoma

- Tumores productores de IGF II 
con hipoglicemia requiere obtener una muestra crítica, es decir durante un episodio de hipoglicemia, para medir concomitantemente glicemia, insulina, hormona de crecimiento, cortisol y T4 libre. Es importante también medir gases en sangre venosa, electrolitos, CPK, amonio, lactato, ácidos grasos libres y cetonemia. Además es aconsejable administrar glucagón durante el episodio de hipoglicemia, ya que junto con corregir la hipoglicemia esta maniobra puede demostrar la presencia de hiperinsulinismo.

La obtención de esta muestra crítica permite orientar el diagnóstico para detectar en forma oportuna cuadros graves, que pueden poner en riesgo la vida del paciente. La figura 1 muestra un algoritmo diagnóstico para la evaluación de la hipoglicemia en la edad pediátrica. Nos parece que todo servicio de Urgencia In- fantil debe estar habituado al diagnóstico y manejo de la hipoglicemia en el niño.

Recalcamos que el manejo de la hipoglicemia es una urgencia tanto diagnóstica como terapéutica, ya que junto con iniciar las medidas para corregir la glicemia y brindar protección al cerebro del paciente, se debe aprovechar la oportunidad para obtener una muestra crítica. Si la muestra crítica no se obtiene durante el episodio de hipoglicemia, se deberá realizar posteriormente una prueba de ayuno para inducir hipoglicemia, con los riesgos asociados para el paciente.

El diagnóstico de estos dos pacientes estuvo basado en la presencia de valores anormales de insulina durante el episodio de hipoglicemia. Posteriormente, se realizó una búsqueda dirigida de una masa en páncreas, ya que esta posibilidad diagnóstica debe ser considerada en

Glucemia menor $50 \mathrm{mg} / \mathrm{dl}$

MUESTRA CRITICA

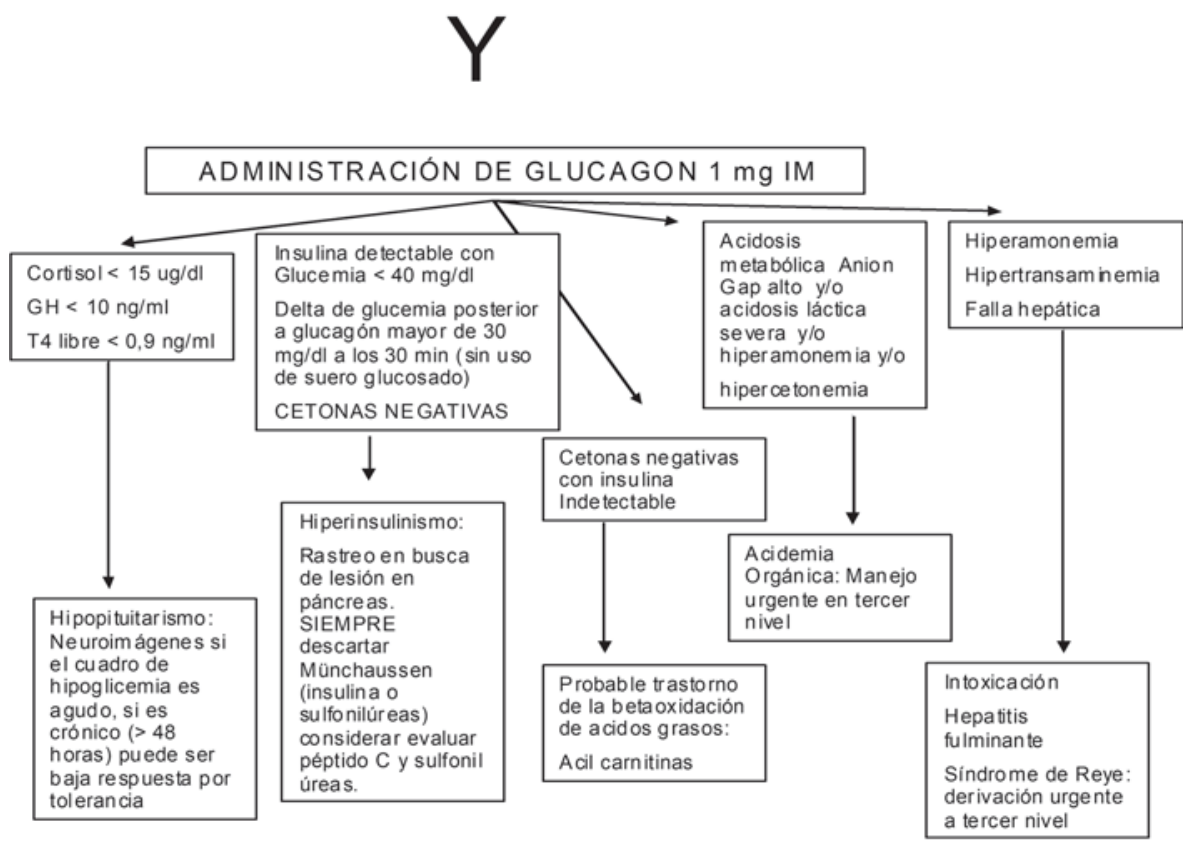

Figura 1. Estrategia diagnóstica de hipoglicemia. 
niños mayores, lo que contrasta con los cuadros de hiperinsulinismo congénito que se presentan durante los primeros meses de vida. En nuestros dos casos el diagnóstico de insulinoma fue confirmado en los estudios por imágenes, sin embargo, la mayoría de las técnicas de exploración para el diagnóstico de insulinoma tiene bajo rendimiento ${ }^{5}$. En estos casos, la laparotomía exploratoria por un cirujano con experiencia en tumores pancreáticos es muy importante y puede detectar la lesión.

La detección de una o varias masas en el páncreas permite plantear el diagnóstico diferencial entre insulinoma esporádico, en el cual se encuentra un adenoma bien diferenciado, con respuesta adecuada al tratamiento quirúrgico con excelente sobrevida; con el síndrome de neoplasia endocrina múltiple tipo 1 (NEM 1) donde se encuentra habitualmente más de una lesión. Estos tumores pueden estar asociados a tumores de paratiroides, adenomas hipofisiarios y se debe vigilar la presencia de síntomas dispépticos por la posibilidad de gastrinoma ${ }^{6}$. En estos casos se debe considerar la posibilidad de alteraciones del gen de la menina para NEM 1 como la causa genética responsable del cuadro de neoplasia endocrina múltiple.

\section{Agradecimientos}

Expresamos nuestros agradecimientos a los Drs. Ricardo Rossi y Ángel Blanco que operaron al primer y segundo paciente respectivamente, y a la Dra. Isabel Torrealba que participó en el manejo del segundo paciente.

\section{Referencias}

1.- Öberg K, Eriksson B: Endocrine tumors of the pancreas. Best Pract \& Res Clin Gastroenterol 2005; 19: 75381.

2.- Gruppuso P, Swartz R: Hypoglycemia in children. Ped Rev 1989; 11: 121-4.

3.- Borras MV, López JP: Diagnóstico diferencial de la hipoglucemia en el niño. Endocrinol Nutr 2005; 53: 493-509.

4.- Tucker ON, Crotty PL, Conlon KC: The management of insulinoma. British J Surg 2006; 93: 264-75.

5.- Tonelli F, Frattini G, Nesi G, et al: Pancreatectomy in multiple endocrine neoplasia type 1-related gastrinomas and pancreatic endocrine neoplasias. Ann Surg 2006; 244: 61-70.

6.- Brandi ML, Gagel R, Angeli A, et al: Guidelines for diagnosis and therapy of men type 1 and type 2. J Clin Endocrinol Metab 2001; 86: 5658-71. 